Aev. Chll. Pedlatr. $64(1) ; 7-13,1993$

\title{
Características de las familias de pacientes reumáticos con y sin corea de Sydenham y de controles sanos
}

\author{
Ximena Berrios C. ${ }^{\text {; }}$ Ingerburg Fuhman V.2; Gloria Sandoval S.' \\ Isabel Salas A. ${ }^{3}:$ Silvia Quiroga D. ${ }^{3}$; Consuelo Alvarado R. ${ }^{4}$
Fandly structure characteristics of Sydenham's chorea patients, rheutnatic fever whithont chorea patients, and a control group

\begin{abstract}
To oblain information about relationships between family sysiem characteristics and rheumatic fever patients with and wilhoul Sydenham's chorea, malched samples of lamilies which included sheumatic potient with (RC) and without chored (R) were compared eoch other and with conirol families no members afected by meumatic fever or any oher chronic diseasel. Appropriate questionaires were individually applied to mother, lather and all siblings of each family group and the fuhrmann.Armengol's famlly graph was applied to the whole family simultaneously. Resulis sugest Ihe presence of an abnomal psychological atmosphere of major instobility within bolh groups of rheumalic potient families in comporisson to controd famities, as meosured by prediclobility $\mid R C 28.8 \%$, R $30.5 \%$, conliols $35.6 \%$. There olso were characteristics of family struclure, among rheumatic patients without chorea, usually with heart disease. that suggest prolective and adaptotise sesponses, for instance higher physical rapprochement rales of sorrow IRC: $25.0 \%, R: 30.5 \%$, controls: $14.8 \%$. Farnily structure may be related to organic diseases like rheumaric fever with or without choreo, in a similar way than in psychosomotic abnomalities. Additional prospective resecich is needed to better understand this relotionship.
\end{abstract}

[Key wordst Sydentram's Chorea, Rheumatic Fever, family structure.]

La historia natural del corea de Sydenham ha sido frecuente tema de debate. Estudios epidemiológicos realizados en Chile y otros lugares sugieren nexos causales de mayor amplitud y complejidad que la infección estreptocócica, tales como hormonales, neurologicos o inflamatorios ${ }^{1:}$. Se ha señalado, también, que el episodio de corea estaría rodeado de una atmósfera de factores psiquicos, sociales, económicos y cultu-

1. Deparamento de Salud Pública, Escuela de Medicina, Universidad Catolica de Chile.

2. Centro de Estudios y Terapia Sistémica de Santiago.

3. Psjeóloga Centro de Estudios y Terapia Sistémica de Santiago.

4. Estadístico. Departamento de Salud Priblica, Escuela de Medicina, Universidad Catolica de Chile.

Fuente de apoyo Grant FONDECYT 87/707. rales adversos y que estos pacientes presentan mayor frecuencia de trastomos emocionales, del rendimiento intelectual, aprendizaje y ajuste social e incluso mayor incidencia de esquizofrenia, después del episodio agudo.10.

Se han encontrado asociaciones entre enfermedad en general y sistema familiar. Inicialmente se postuló que había influencia recíproca entre enfermedad psicosomática y estructura del sistema familiar; posteriormente se describieron algunas características recurrentes como gran preocupación de cada uno por los demás, con importante involucración mutua y limitación del desarrollo personal y de los subsistemas familiares. Se han descrito, por ejemplo, algunas peculiaridades en familias donde un hijo sufre de asma, problemas conductuales, retardo mental o trastomos específicos del desarrollo. Se ha sugerido que las ca- 
racterísticas descritas para familias con problemas psicosomáticos, también podrían estar presentes en otras con dificultades evidentemente orgánicas, destacando entre esas peculiaridades, distancias emocionales entre los conyuges, desigualdad en las de ambos padres con sus hijos y mayor o menor importancia relativa del hijo con problemas respecto de sus hermanos. Estas características parecen más relacionadas con el significado de la enfermedad para la familia, que con ia especificidad del problema ${ }^{11-19}$.

La relación entre estructura familiar y enfermedad permite proponer la hipótesis de diferencias en la estructura de las familias con pacientes reumáticos con o sin corea de Sydenham, que podrian guardar más relación con la gravedad de la enfermedad o la importancia para la familia, que con el diagnóstico específico mismo. Una diferencia entre la enfermedad reumática sin corea y el corea de Sydenham aislado, permitiría aumentar la certeza que se trata de dos entidades nosológicas distintas. La presencia de aspectos psicológicos de la dimensión familiar, peculiares de la enfermedad reumática o del corea de Sydenham aporta nuevas altemativas de prevención secundaria. El presente estudio aporta información para este enfoque del problema.

\section{Material y Método}

Se disejó un estudio de muestras equivalentes para la comparación de familias en que uno de $\mathrm{krs}$ miembros había sufrido corea de Sydenham (grupo de corea), otro (grupo de enfermedad reumática) de familias que contaban con algún paciente con enfermedad reumática inactiva al momento dcl estudio, pero que nunca habia prescntado corea de Sydenham y orro de familias conirol. Los pacientes del grupo de corea, actualmente inactivos, debían haber presentado uno o más episodios de cones de Sydenham durante los úlimos cinco años y estar enrolados en el programa de control y prevención de enfermedad reumática en el Servicio de Salud Meiropolitano Sur Oriente (SSMSO). Los pacientes del grupo de enfermedad reumática debían tener antecedentes de carditis a poliartritis en los úlímos años, o evidencia de valvuloparía reumática al momento de ingreso al estudio, participando, igualmente, del programa de preveneión mencionado. El grupo control formado por familias en que nínguno de sus miembros tenía antecedentes de enfermedad reumática, corea de Sydenham u oura enfermedad crónica fue reclutado de la población general del S\$MSO.

La muestra fue parcada tomando como referencia cada caso índice del grupo corea, considerando variables sexo, edad, situación econónica, ubicación geográfica, tamaño y constitución del grupo familiar. Esto se evaluó con ayuda de un instrumento estandarizado que inclnyó la encuesta de Graffar que define el nivel sociceconomico ${ }^{20}$. La mayor pane de la información fue recogida en el domicilio de las familias elegidas. La rigurosa configuración de los grupos para una adeciada comparabilidas, obligó a desechar un número importante de encuestas y entrevistas, finalizando el estudio con 18 familias en cada uno de los grupos esiudiados.

La recolección de información acerca del sistema familiar fue hecha por psicólogos de una unidad de neurología, utilizando un cuestionario diseñado por terapeutas familiares del "Centro de eswudios y terapia sistémica de Santiago", quienes adaptaron un cuestionario aplicado en $1 \mathbf{r a -}$ bajos anteriores 5 . 16 . Este contierle preguntas acerca de cohesión y diferenciación del sistema familiar; configuración límiles y desarrollo interno de los subsistemas y estabijdad de la organización. Se aplicó una versión del cuestionario a los padres y otro a los hijos y la gráfica familiar de Fuhrmann-Amengolls, 16, inspirado en los trabajos de $S$. Thrower ${ }^{3 l}+$ al grupo familiar total. Este instrumento evalúa variables como distancia emocional e importancia relativa de cada uno de los integrantes de la familia y, a través de la superposición, la permeabilidad de los límites interpersonales.

La aplicación de los cuestionarios y de la gráfica familiar fue hecha en los domicilios, donde debían estar presentes todos los integrantes simultáncamente, para la construceión conjunta de dicha grálica. Sc tuvo cuidado de asegurar la aplicacion en ciego para evitar la contaminación de expecjativas de Jos evaluadores en los resultados.

La distribución de cada variable de las encuestas en los tres grupos se snalizó mediante el ensayo de chi cuadrado o la comparación de proporciones y la ínformación -traducida a dígitos (digitalizada)- de la grâfjca familiar, mediante an álisis de varianza y el ensayo de Turkey. Se trabajó con un rivel de significación estadística de $\mathrm{p}<0,05$. La información fue procesada en el Depariamente de Salud Pública de la Universidad Carólica de Chile.

\section{Resultados}

Se logró aplicar todos los instrumentos disenados para el estudio a 18 farmilias de los tres grupos investigados, cuyos integrantes sumaron 326 personas encuestadas. Sus características, según las variables de pareamiento, se encuentran en la tabla 1 , que muestra la alta comparabilidad de las muestras en las variables que se pretendía concralar.

Las características que discriminaron entrc los tres grupos estudiados o entre dos de ellos, fueron las siguientes:

Involucración intrafamiliar para reducir el conflicto. Los hijos del grupo de corea se involucran, más que los restantes, en los conflictos 
Tabla 1

Caracteristicas de las familias incluidas en el estudio, según casos índice, padres, hijos y situación socioeconómica

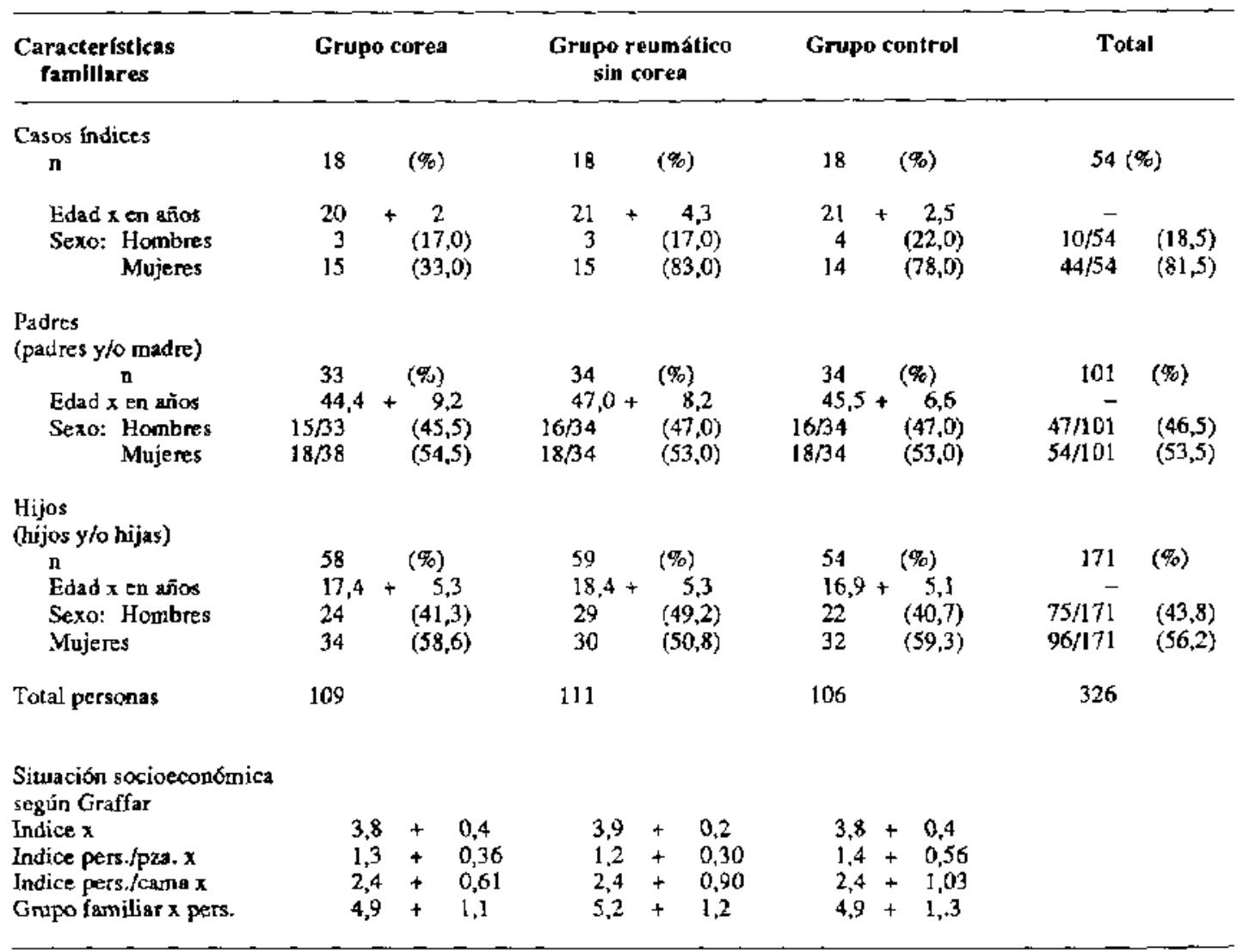

de los miembros de su familia, para atenuar los desacuerdos entre ellos: $88,5 \%$ vs $66,7 \%$ y $86,0 \%$ en los grupos de enfermedad reumática y control respectivamente (diferencia significativa entre grupo corea y grupo reumático $p<0,05)$.

Cercanía afectiva. Los sentimientos de tristeza de otro (tabla 2) acercan más a los hijos de las familias de grupo de enfermedad reumática que de los otros dos grupos. El sentimiento de alegría, en cambio (tabla 3) los acerca menos que la tristeza, a diferencia de los hijos de controles en que la alegría los acerca más que los sentimientos de tristeza. Los padres del grupo reumático son más sensibles ante los sentimientos de alegría y de tristeza que los padres de los otros dos. La diferencia se hace significativa cuando el sentimicnto del otro es la alegria.

La jerarqula parental en las decisiones. La jerarquía parental está más claramente definida en las familias de reumáticos que en los otros dos grupos, lo que se expresa en la percepción de los hijos que se consideran más obedientes ante los mandatos de sus padres: $30,5 \%$ vs $23,2 \%$ y $14,8 \%$ en los grupos corea y control respectivamente, siendo la diferencia estadísticamente significativa $(p<0,05)$ entre reumáticos y controles.

Tiempo libre comparido. Los padres del grupo de enfermedad reumática comparten mayor tiempo libre con su familia que los otros dos grupos. A la altenativa "mucho tiempo", respondieron afirmativamente $91,2 \%$ de los padres, 
Tabla 2

\begin{abstract}
Cercanía afectiva ante tristeza según grupo estudiado. Respuesta de acercamiento de los hijos ante tristeza de otro miembro familiar
\end{abstract}

\begin{tabular}{lccc}
$\begin{array}{l}\text { Forma de } \\
\text { acercamiento }\end{array}$ & $\begin{array}{c}\text { Grupo corea } \\
\text { "Co" }\end{array}$ & $\begin{array}{c}\text { Grupo enfermedad } \\
\text { reumfitica } \\
\text { "R" }\end{array}$ & $\begin{array}{c}\text { Grupo } \\
\text { control } \\
\text { "Ct" }\end{array}$ \\
\hline & $\mathrm{n}=56$ & $\mathrm{n}=59$ & $\mathrm{n}=54$ \\
Fisico & $25,0 \%$ & $30,5 \%$ & $14,8 \%$ \\
Verbal & $58,9 \%$ & $62,7 \%$ & $68,5 \%$ \\
\hline Total & $83,9 \%$ & $93,2 \%$ & $83,3 \%$ \\
\hline
\end{tabular}

Difercncia entre " $\mathrm{R} " / \mathrm{Ct}$ " y " $\mathrm{R}$ " $/ \mathrm{C}$ " $\mathrm{C}$ " significativas $\mathrm{p}<$ 0.05.

\section{Tabla 3}

Acetcamiento físico por trisleza y por alegría. según grupo estudiado. Acercamiento físico de los hijos y padres ante sentimientos de tristeza y de alegría de otro miembro familiar.

\begin{tabular}{lccc}
$\begin{array}{l}\text { Sentimlento } \\
\text { que acerca }\end{array}$ & $\begin{array}{c}\text { Grupo corea } \\
\text { "Co" }\end{array}$ & $\begin{array}{c}\text { Grupo enfermedad } \\
\text { reumatica } \\
\text { "R" }\end{array}$ & $\begin{array}{c}\text { Grupo } \\
\text { centrol } \\
\text { "Ct" }\end{array}$ \\
\hline Hijos & $\mathbf{n}=56$ & $\mathrm{n}=59$ & $\mathrm{n}=54$ \\
Tristeza & $25,0 \%$ & $30,5 \%$ & $14,8 \%$ \\
Alegria & $28,6 \%$ & $23,7 \%$ & $33,3 \%$ \\
Padres & $\mathbf{n}=33$ & $\mathrm{n}=34$ & $\mathbf{n = 3 4}$ \\
Tristeza & $46,9 \%$ & $47,1 \%$ & $41,2 \%$ \\
Alegria & $30,3 \%$ & $52,9 \%$ & $29,4 \%$ \\
\hline
\end{tabular}

Diferencia hijos: Tristeza/Alegría en "Cr" significativas $p<$ 0,05 .

Diferencia padres: "R" $\mathrm{R}^{\prime \prime C l} " \mathrm{p}<0,05$ y "R" $/ \mathrm{Co}$ " $\mathrm{p}<0,01$

versus $67,6 \%$ y $66,7 \%$ en control y corea respectivamente, la diferencia es significativa $(p<0,05)$ entre el gnupo reumático y los otros dos.

Previsibilidad del sistema familiar: Esta variable puede ser considerada como un indicador de estabilidad de un sistema, puesto que a mayor repetición de secuencias conductuales, mayor es la probabilidad de predecir correctamente los hechos. Las respuestas de los padres como las de los hijos refieren una menor precisión para predecir las conductas de los demás en los grupos corea y reumático que en grupo control (tabla 4). La diferencia descrita es más evidente aún al analizar sólo las respuestas de los padres, quie- nes tienen más aciertos de predicción cuando pertenecen a las familias del grupo control, con $97,0 \%$ vs $75,8 \%$ y $73,5 \%$ en los grupos reumấticos y corea, respectivamente. La diferencia es estadísticamente significativa $(p<0,01)$ entre los grupos reumáticos y control y $(\mathrm{p}<0,05)$ entre los grupos corea y control.

Adhesion. La percepción de "ser escuchado" por los demás, asociada a cercania afectiva en investigaciones anteriores (comunicacion a las II Jornadas de Terapia Familiar, por uno de los autores, Santiago 1989), fuc mayor en los padres del grupo control $(88,2 \%)$, que en grupo reumático $(72,7 \%)$ y es francamente menor $(63,6 \%)$ en cl grupo corea, siendo estadísticamente significativa $(p<0,05)$ la diferencia entre control $y$ Corea.

Superposición de los círculos individuales en la gráfica familiar. En el grupo control sólo $7,1 \%$ de las familias presentan una superposición de 0,20 a 1 de la superficie de sus círculos. Este porcentaje se triplica en el grupo de corea y es seis veces mayor en el grupo enfermedad reumática (tabla 5). Esta característica concuerda con las proporciones de S. Thrower ${ }^{21}$ quien utiliza su "círculo de la familia" para representar la "aglutinación, propia de las familias con enfer" medad psicosomáticas" mediante la superposición de los círculos individuales.

\section{Comentario}

Este estudio presentó varias dificultades metodológicas. El pareamiento riguroso de los grupos poblacionales hizo más lento y costoso el proceso de su conformación. La aplicación de la grafica familiar, que exigía la colaboración simultánea de toda la familia, contribuyó a la complejidad y dificultad en su realización. El análisis mismo también resultó complejo y demoroso, ya que hubo necesidad de crear nuevas variables y traducir a dígitos los datos de la grafica familiar.

En lo referente a las características del sistema familiar, algunas configuraciones descritas en la enfermedad psicosomática, estuvieron presentes en forma alternada, tanto en el corea de Sydenham como en la enfermedad reumática.

En las familias de pacientes con corea de Sydenham los hermanos se esfuerzan por atenuar conflictos, hay mayor distancia interpersonal y 


\section{Tabla 4}

Previsibilidad de comportamientos

del resto de la familia, según grupo estudiado. Opinión de padres y de hijos.

\begin{tabular}{|c|c|c|c|}
\hline $\begin{array}{l}\text { Sentimiento } \\
\text { que acerca }\end{array}$ & $\begin{array}{c}\text { Grupo corea } \\
\text { "Co" }\end{array}$ & $\begin{array}{l}\text { Grupo enfermedad } \\
\text { reumstlica } \\
\text { "R" }\end{array}$ & $\begin{array}{l}\text { Crupo } \\
\text { control } \\
\text { "Ct"" }\end{array}$ \\
\hline $\begin{array}{l}\text { Alia } \\
\text { Mediana } \\
\text { Baja }\end{array}$ & $\begin{array}{l}28,8 \% \\
51,3 \% \\
20,0 \%\end{array}$ & $\begin{array}{l}30,5 \% \\
40,0 \% \\
29,5 \%\end{array}$ & $\begin{array}{l}35,6 \% \\
54,0 \% \\
10,3 \%\end{array}$ \\
\hline$N^{2}$ total & $n=80$ & $n=95$ & $n=87$ \\
\hline
\end{tabular}

Chi-cuadrado $=10,99 ;$ g.l. $=4 ; p<0,05$.

Diferencis ente " $\mathrm{Ct} / \mathrm{r}$ " $\mathrm{R}$ " es significativa $\mathrm{p}<0,0 \mathrm{t}$.

\section{Tabla 5}

Superposición de círculos en la gráfica familiar, según grupo estudiado.

\begin{tabular}{lccc}
$\begin{array}{l}\text { Superposiclon Grupo corea Grupo enfermedad } \\
\text { mayor de 0,20 } \\
\text { (rango 0-1) }\end{array}$ & "Co" & $\begin{array}{c}\text { Grupo } \\
\text { reumatica } \\
\text { "R" }\end{array}$ & $\begin{array}{c}\text { control } \\
\text { "Ct" }\end{array}$ \\
\hline $\mathrm{n}=14$ & $\mathrm{n}=17$ & $\mathrm{n}=14$ \\
$\mathrm{Alta}$ & $21,4 \%$ & $41,2 \%$ & $7,1 \%$ \\
\hline
\end{tabular}

Las diferencias son significativas entre " $\mathrm{R}^{*} /{ }^{\mu} \mathrm{Cl}$ " en nivel de $\mathrm{p}<0,05$.

menor estabilidad del sistema. Estas caracteristicas sugieren una "atmósfera de peligro, soledad e inestabilidad" en que los hijos son los mayores garantes de su sistema familiar, pucs, los padres se activan menos que ellos en pro de la armonía familiar. En trabajos anteriores no se habia descrito una "atmósfera psicológica particular", de la familia del paciente reumático habitual, con secuela cardíaca. La mayor cercanía afectiva que sugieren estos resultados podría ser interpretada como una respuesta adaptativa del sistema familiar ante el peligro en que se encuentra uno dc sus integrantes. En este contexto es esperable mayor cercanía en la familia del paciente con valvulopatía reumática que con antecedente de corea, dada la connotación más alarmante que suele tener una enfermedad del corazon. Del mismo modo podría comprenderse que en las familias del grupo enfermedad reumática (sin Corca) se comparta más el tíempo libre que en los otros dos, lo que también apuntaría a una mayor protección mutua. Finalmente, tampoco es de extrañar que en un sistema "en emergencia" (menor estabilidad) como sería la familia del paciente reumático, se defina la jerarquía más claramente que en otro, cuya integridad no se vea tan amenazada. Algunas de estas características pueden tener un efecto adaptativo: mayor cercanta permite mejor enfrentamiento de dificultades (cercanía ante tristeza); más tiempo compartido aumenta la protección mutua; la jerarquia parental clara, protege a un sistema expucsto a riesgo de complicaciones de una enfermedad temible.

Los resultados de cste estudio comparativo definen algo más que la atmósfera especial que rodea al paciente con corea de Sydenham. Es un hallazgo novedoso e interesante, descubrir que el ambiente del paciente reumático sea quizás más especial que el de corca. Las características descritas, por ejemplo, la solidaridad, la renuncia personal y disposición de ayuda, explican que esa atmósfera particular del paciente rcumático haya pasado desapercibida. Los miembros de la familia del paciente reumático parecen cxistir el uno para ei otro, lo que es cómodo para el médico y su acción tcrapética específica de prevención secundaria de la enfermedad cardíaca. Cabría preguntarse si la disposición a cumplir las indicaciones y la responsabilidad en los controles no tendrá repercusiones en otras áreas del sistema familiar, que pudieran influir ncgativamente en el paciente.

La única variable que diferencia el grupo control de Jos otros dos grupos, es la previsibilidad del funcionamiento familiar, to que sugiere que ambas enfermedades podrían tender a desestabilizar la organización familiar, inestabilidad que se mantendría mientras persista la enfermedad o el temor a las repercusiones de ella.

Dada la complejidad de los fenómenos psiquicos es difícil encontrar variables aisladas, que por sí solas tengan el peso suficiente para discriminar, especialmente tratándose de muestras pequenas de familias. De este problema proviene la necesidad de buscar formas de análisis más complejos, que eliminen la direccionalidad de las determinaciones y den cuenta de la complejidad de las redes causales, to que exige a su vez el estudio con muestras de gran tamaño, difíciles de obtener debido a los problemas mencionados en la recolección de la información.

El número reducido de variables que discriminaban significativamente entre los grupos es- 
tudiados, además del tamaño de la muestra, puede tener que ver también con nuestro excesivo celo al parear las muestras del estudio. Es posibJe que la rigurosidad no llevara a parear variables (por ejemplo, aspectos del nivel de vida o del tamato o composición del sistema familiar) relacionados de algún modo, desconocido para nosotros, con la patología. Otro aspecto que podría tener importancia, es que los pacientes del grupo reumático, tienen frecuentemente secuelas cardíacas, lo que mantiene la preocupación por la salud a través del tiempo, y puede repercutir en el funcionamiento de la familia. Esto no sería tan importante en el grupo de pacientes con corea de Sydenham, quienes en su mayoría son puros y la preocupación es menos persistente, ya que el corea en sí, no produce secuela evidente. Este hecho podría explicar las diferencias entre las farnilias de los pacientes reumáticos y los coreicos y las similitudes entre las familias con paciente coreico y del grupo control.

Los resultados abtenidos sugieren que la cnfermedad y la organización familiar se infuyen reciprocamente. Esta relación mutua puede configurar un círculo beneficioso en que la adaptación del sistema familiar reduce el riesgo, la duración o la gravedad de la enfermedad. Podría crearse también un círculo vicioso, en que los mecanismos habitualmente adaptativos pudieran significar un riesgo para el sistema familiar, en que al reducirse, por ejemplo, el desarrollo personal, pueden aparecer problemas que indirectamente se vuelven en contra del supuestarnente beneficiado. Nuestro supuesto, que toda la enfermedad puede modificar la organización y la estructura del sistema familiar, concuerda con la ausencia de un perfil caracteristico de la familia en que hay un paciente con corea de Sydenham, puesto que hemos postulado que las características organizacionales de la familia que acompañan a una enfermedad en particular son inespecíficas y tengan de este modo una mayor relación con la gravedad y su significado para la familia que con el diagnóstico médico.

Dirigiendo la atención al grupo familiar en que el paciente reurnático o de corea se encuentra inserto y teniéndolo en cuenta, más allá de su colaboración en el cumplimiento de las indicaciones, es posible que el especialista pudiera ser más efectivo, al integrar a todo el grupo familiar en sus acciones preventivas.

\section{Resumen}

Con el propósito de aportar información sobre la relación de la estructura familiar con la enfermedad reumática con o sin corea de Sydenham, se disefió un estudio de muestras equivalentes pareadas de familias de pacientes con corea, de pacientes reumáticos sin corea y de familias controles sin antecedentes de enfermedades cronicas entre sus miembros. Todos los padres, madres y hermanos de los casos índice de cada familia fueron encuestados por separado mediante un cuestionario especialmente preparado. La gráfica familiar de Fuhrmann-Armengol se aplicó a todos los componentes de cada familia simultáneamente. Los resultados indican una atmósfera psicológica de mayor inestabilidad en los dos gtupos de pacientes que en el grupo control, pues, la previsibilidad del sistema farniliar. cuando es alta $(28,8 ; 30,5$ y $35,6 \%)$ o mediana $(51,3 ; 40,0$ y $54,0 \%)$-respectivamente en corea, reumáticos y controles- es siempre mayor en los últimos. Se observan características estructurales de las familias con pacientes reumálicos sin conea, especialmente con cardiopatía, que sugieren una respuesta protectora adaptativa de este sistema familiar, pues, la cercanía afectiva de los hijos ante el sufrimiento de otro integrante es del orden de $93 \%$ en contraste con $83,9 \%$ en Ias familias de pacientes con corea y $83,3 \%$ en controles, como también en la cercanía ante la tristeza y la alegría donde las correspondientes proporciones fueron 30,5 y $52,9 \%$ respectivamente, en reumáticos, versus 25 y $30,3 \%$ en familias con corea, así como 14 y $29,4 \%$ en controles. Se concluye que la estructura familiar, no sólo se relaciona con la enfermedad psicosomática, sino que también con enfermedades orgánicas como la enfermedad reumática con y sin corea de Sydenham. Es necesario hacer más investigación para comprender mejor estas asociaciones.

(Palabras clave: corea de Sydenham, enfermedad reumática, estructura familiar).

\section{Referencias}

1. Berrios $X, Q$ wesney $F$, Morales A, Blazquez $J$, Lagomarsino $E, B$ isno A: Acute Rheumatic Fever and Poststreptococcal. Glomenulonephritis in an open population: Comparative studies of epidemiology and bacteriology. J Lab Clin Mad 1986; 108: 4, 35-42. 
2. Berrios $X$, Morales A, Guzmán B, Quesney $F$. El corea de Sydenham en el Servicio de Salud Metropolitano Sur Oriente: Descrípción epidemiológica, Rev Med Chile 1987; 115; 470-476.

3. Berrios X. Quesnzy F. Morales A, Blazquez J, Bisno A: Are all recurences of "Pure" Sydenham Chorea true recurrences of Acute Rheumatic Fever? I Pediatr 1985: 107 : $867-872$.

4. Berrios X: El Corea de Sydenham recidivante: Una extrafía manifestación de Enfermedad Reumática. Rev Med Chile 1986; 114: 254-256.

5. Taranta A: Relation of isolated recurrences of Sydenham's chorea to proceding streptococcal infections. N Engl J Med 1959; 260: 1204-1210.

6. Jonas $S$, Spagnuolo M, Xloth $H H$ : Chorea gravidanum and streptococal infection. Obstet Gynecol 1972; 39: $77-79$.

7. Riddoch $D$, Jefferson $M, B$ ickersLaff ER: Chorea and the oral contraceptives. Br Med J 1971; 4: 217-218.

B. Herd $J K$, Medhi $M$, Unendoski DM: Chorea associated with Systemic Lupus Erythematosus: report of two cases and review of the literature. Pedialrics 1978; 61: 308-315.

9. Aron AM, Freeman $M M$. Carter $S$ : The natural history of Sydenham's Chorea: review of the literature and longtem avaiuation with emphasis on cardiac sequelae. Am 5 Med 1965: 38: 83.95 .

10. Nausieda PA, Grossman BJ. Koller WC et al.: Sydenharn's Chorea: un update. Neurology 1980; 30: 331-334.

11. Minuchin S: Conceptual Model of Psychosomatic
Illness in Children. Arch Gen Psychiat 1975; 32: 77 79.

12. Minuchin S, Rosman B, Baker $I$ : Psychosomatic Families. Harvard University Press, Cambridge Mass. USA, 1978.

13. Minuchin $S$ : Families and Family thesapy. Harvard University Press, Cambridge Mass. USA, 1974.

14. Minuchin S: Técnicas de Terapia Familiar. Ed. Paidos, Buenos Aires, Argentina, 1984.

15. Fuhrmann 1, Armengol V: Est ructura y Diagrama Familiar. 1) Estnuctura familiar. Una Modalidad Diferente para Explorar. Sistemas farniliares 1992; 8: 39-44.

16. Fuhmann 1. Armengol $V$, Labarco AM: Estructura y diagrama familiar. 2) Interrclación de variables estruclurales. Sistemas familiares 1992; 8: 45-53.

17. Fuhrmann I, Armengol $V$ : Análisis sistémico de la familia chilena. Revista de Eđucación 1988; 160: 52-56.

18. Fuhrmann $I$, Chadwick $M$ : Taller para padres, una red de apoyo para niños que tienen problemas escolares. Revista de Edacación 1992; en prensa.

19. Chadwick $M$, Fuhrmonn I: Una incorporación de apoderados en la rehabilitación integral de niños con trastomos específicos. Lectura y Vida 1991: 9: 15-22.

20. Gómez M, Questey F, Kirschboum A, Lopez I: Estudio de la situación socioeconómica de la población de un real de salud. Tesis para optar al grado de Especialista en Salud Pública, Escuela de Medicina, Universidad Católica de Chile, 1984.

21. Thrower $S$, Bruce $W, W a l t o n ~ R$ : The family circle method for integraling family. System concepts in family medicine. The J Fam Practice 1982; 15: 451-457. 